\title{
Correction: Magic roundabout is an endothelial-specific ohnolog of ROBO1 which neo-functionalized to an essential new role in angiogenesis
}

\section{Lukasz Huminiecki}

There are errors in the Funding statement. The correct Funding statement is as follows: LH was funded to perform this work by the National Science Centre, Poland, grant POLONEZ 2 (grant number 2016/21/P/NZ2/03926). This project has received funding from the European Union's Horizon 2020 research and innovation programme under the Marie SkłodowskaCurie grant agreement No. 665778. The funders had no role in study design, data collection and analysis, decision to publish, or preparation of the manuscript.

\section{Reference}

1. Huminiecki L (2019) Magic roundabout is an endothelial-specific ohnolog of ROBO1 which neo-functionalized to an essential new role in angiogenesis. PLoS ONE 14(2): e0208952. https://doi.org/10.1371/ journal.pone.0208952 PMID: 30802244

G open ACCEss

Citation: Huminiecki L (2020) Correction: Magic roundabout is an endothelial-specific ohnolog of ROB01 which neo-functionalized to an essential new role in angiogenesis. PLOS ONE 15(3): e0230384. https://doi.org/10.1371/journal. pone.0230384

Published: March 6, 2020

Copyright: @ 2020 Lukasz Huminiecki. This is an open access article distributed under the terms of the Creative Commons Attribution License, which permits unrestricted use, distribution, and reproduction in any medium, provided the original author and source are credited. 